\title{
ISOLATION OF TALAROMYCES FLAVUS FROM ROODEPLAAT DAM AND SCREENING OF ITS SECONDARY METABOLITES IN ARTIFICIAL MEDIA
}

\author{
MagWAZA, N. M. ${ }^{1}$ - NXUMALO, E. N. ${ }^{1}-$ MAMBA, B. B. ${ }^{1}-$ NYONI, H. ${ }^{1}-$ NTUSHELO, K. $^{2}-$ \\ MSAGATI, T. A. M. ${ }^{*}$ \\ ${ }^{1}$ University of South Africa, College of Science Engineering and Technology, Institute for \\ Nanotechnology and Water Sustainability, UNISA Science Campus, Florida Park, 1710 \\ Roodepoort, South Africa \\ ${ }^{2}$ University of South Africa, College of Agriculture and Environmental Sciences, Department of \\ Agriculture and Animal Health, UNISA Science Campus, Florida Park, 1710 Roodepoort, South \\ Africa \\ *Corresponding author \\ e-mail: msagatam@unisa.ac.za \\ (Received $12^{\text {th }}$ Jun 2019; accepted $25^{\text {th }}$ Oct 2019)
}

\begin{abstract}
This paper reports on a divergent approach employing isolation methods and high-resolution mass spectrometry to screen decomposition products that are a result of fungal action on biomass present in aquatic media. Fungi decompose organic matter in surface water through a process which plays a major role in nutrient cycling and maintenance of aquatic life. Moreover, the presence of pathogenic or potentially pathogenic species of fungi in drinking water distribution systems, may cause chronic diseases to consumers and these pathogenic fungi have the potential to produce a variety of secondary metabolites which play important roles in protecting the organism against competition for nutrients and survival. In this paper, the fungus Talaromyces flavus was isolated from Roodeplaat Dam in Gauteng province (South Africa) and was identified using sequence analysis of the internal transcriber spacer (ITS) region. Secondary metabolites produced by $T$. flavus grown in liquid culture were extracted and determined using Liquid chromatography-mass spectrometry (LC-MS) and comparative analysis of the secondary metabolite profile was done using Aspergillus fumigatus as a reference (ATCC 36607). The influence of nutrients, $\mathrm{pH}$ and incubation period on the production of secondary metabolites was demonstrated and validated statistically by principal component analysis (PCA).
\end{abstract}

Keywords: fungi, mycotoxins, LC-QTOF-MS, molecular techniques, aquatic life, PCA

Abbreviations: ITS: internal transcriber spacer; LC-MS: Liquid chromatography-mass spectrometry; PCA: principal component analysis

\section{Introduction}

Freshwater fungi produce a diversity of antimicrobial metabolites, which help them compete against other microorganisms (Calvo et al., 2002; El-hasan et al., 2009; Connor et al., 2016). However, some of them produce compounds which are responsible for inflammation and chronic diseases (Hernández-Carlos and Gamboa-Angulo, 2011). Numerous groups of secondary metabolites and bioactive compounds have been isolated from fungal strains collected from diverse environments (Zhao et al., 2010; Swathi, 2013; Imhoff, 2016). A wide range of natural products produced by a variety of fungal species are used for medication, industrial and agricultural purposes (Khan et al., 2014). Some of these compounds are deleterious, while others are beneficial to humankind.

The production of secondary metabolites can be affected by the $\mathrm{pH}$ and nutrient levels in the aquatic environment such as carbon and nitrogen sources (Singh et al., 
2010; Schulthess et al., 2014). The manipulation of growth conditions can lead to the production of various compounds, since most of the natural products are secreted by microbes under specific sets of conditions. The physical parameters such as $\mathrm{pH}$, nutrients, temperature and incubation period can be monitored for the production of diverse structures of compounds for therapeutic uses (Gaden, 2000). The objectives of the study is to investigate the influence of nutrients in the dam water and nuclease-free water by spiking the water sample with isolated fungal Talaromyces flavus and the other set with the reference strain of Aspergillus fumigatus (ATCC 36607). As well as screening and profiling of the secondary metabolites produced by aquatic fungi in water, using liquid chromatography coupled to quadrupole/time-of-flight mass spectrometry (LC-Q-TOF-MS/MS) and SIMCA software.

\section{Materials and methods}

\section{Water sampling}

The water samples were collected in November 2015 at Roodeplaat Dam in Gauteng, South Africa. The wall of the dam is $55 \mathrm{~m}$ high and has a length of $351 \mathrm{~m}$. Two main sampling sites were selected within the dam, and these were $50 \mathrm{~m}$ apart; at each site the samples of water were collected in $1 \mathrm{~L}$ clean uninfected bottles at $5 \mathrm{~m}$ apart. Two $1 \mathrm{~L}$ water samples were selected from the samples points 40 and 50 as indicated in Figure 1. These sampling points represent bigger part of the dam. Therefore, water samples were examined for physical and chemical properties such as $\mathrm{pH}$, electric conductivity, temperature and nitrates using a multiprobe (YSI professional plus multiparameter water quality instrument, built in barometer, BOD sensors includes 2-meters cable; 1 or 4 meters on lab $\mathrm{pH}, \mathrm{ORP}$ and $\mathrm{pH} / \mathrm{ORP}$ cables, four electrode cells).

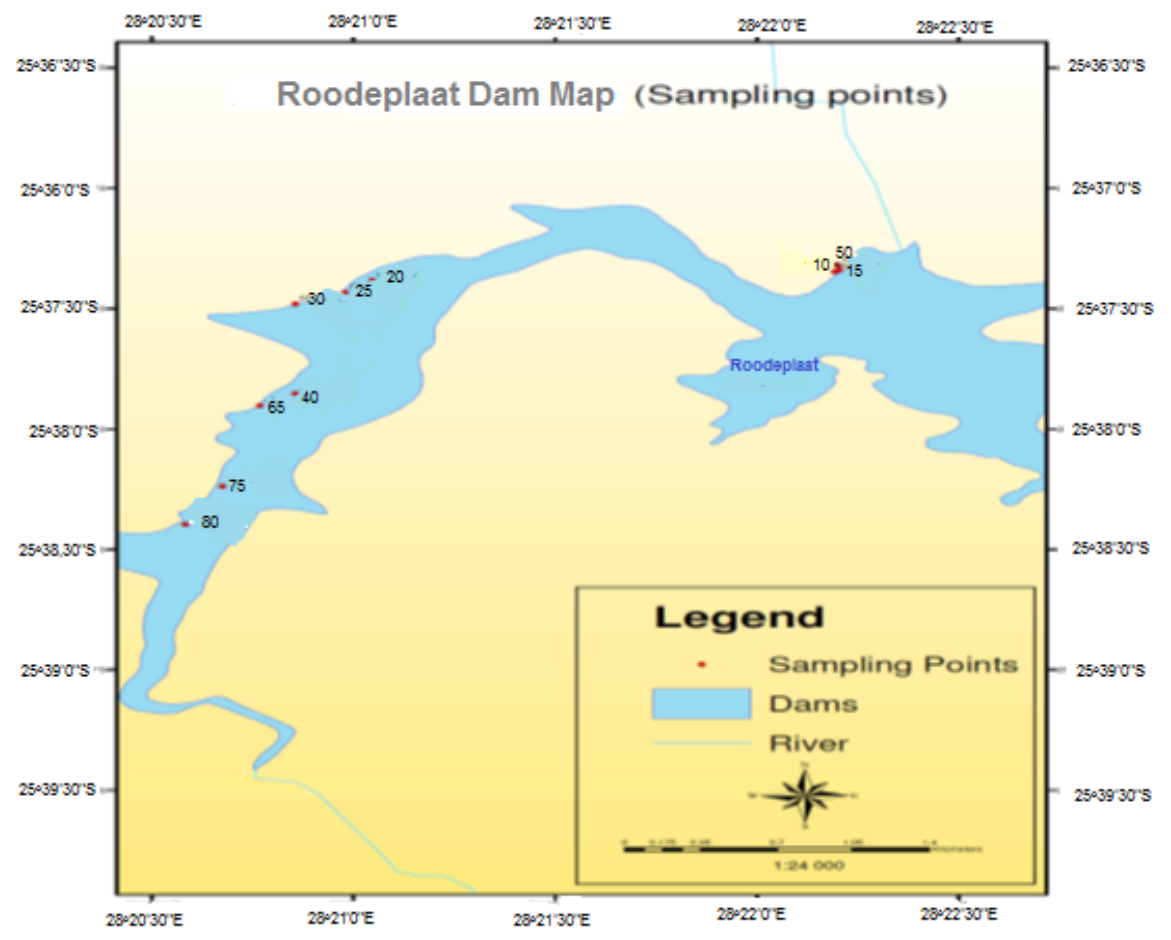

Figure 1. The sampling points in Roodeplaat Dam 


\section{Fungal cultures and DNA extraction}

Isolation of fungi was obtained through the pour-plate method; using a pipette, about $200 \mu \mathrm{L}$ of dam water was spread evenly over the surface of three culture plates of potato dextrose agar (PDA) (Sigma-Aldrich, product number P2182). The culture plates were incubated at $25 \pm 2{ }^{\circ} \mathrm{C}$ for 2 weeks. Cultures were visually examined for colony growth and colour daily. Fungal colonies were examined and then transferred to freshly prepared PDA supplemented with streptomycin sulphate using the hyphal tipping method. The new cultures were then incubated at $25 \pm 2{ }^{\circ} \mathrm{C}$. Sub-culturing of mycelia cut from the colony edges continued until pure colonies were obtained. One fungal colony was selected for identification and further study.

\section{DNA extraction}

The selected fungal colony was ground with liquid nitrogen using a mortar and pestle and DNA was extracted from 1gram mycelium powder of five-day-old pure fungal cultures using the QIAamp ${ }^{\circledR}$ DNA mini kit (Qiagen). The total quantity of genomic DNA extracted was quantified using the Quantus ${ }^{\mathrm{TM}}$ Fluorometer (Promega, USA).

\section{Polymerase chain reaction}

Polymerase chain reaction (PCR) was used to amplify the ITS region using the universal primer pair, ITS1F (5'CTTGGTCATTTAGAGGAAGTAA3') and ITS4 (5'TCCTCCGCTTATTGATATGC3'), obtained from Inqaba Biotechnologies, Pretoria, South Africa. The total reaction volume of $25 \mu \mathrm{L}$ consisted of $1.0 \mu \mathrm{L}$ of ITS1 forward primer, $1.0 \mu \mathrm{L}$ of ITS4 reverse primer, $12.5 \mu \mathrm{L}$ of DreamTaq Green PCR Master Mix (GoTaq ${ }^{\circledR}$, Green Master Mix, Promega, USA), $3 \mu \mathrm{L}$ of DNA (template more than $50 \mathrm{ng}$ ), and $7.5 \mu \mathrm{L}$ of nuclease-free water. The PCR setting were as follows, denaturation step of $94{ }^{\circ} \mathrm{C}$ for $5 \mathrm{~min}$, proceeded by 35 cycles which were made up of denaturation at $94{ }^{\circ} \mathrm{C}$ for $1 \mathrm{~min}$, annealing at $57^{\circ} \mathrm{C}$ for $30 \mathrm{~s}$, extension at $72{ }^{\circ} \mathrm{C}$ for $30 \mathrm{~s}$, lastly was elongation stage of $72{ }^{\circ} \mathrm{C}$ for $10 \mathrm{~min}$. The reaction was finally terminated at $-4{ }^{\circ} \mathrm{C}$. Upon completion of the PCR run, a product of $5 \mu \mathrm{L}$ was loaded on a $1 \%$ agarose gel for electrophoresis to confirm the amplification of the target \pm 500 base-pair fragment in the ITS region. The remainder of the PCR sample was submitted for sequencing at Inqaba Biotechnologies (RSA, Gauteng, Pretoria). The sequence analysis chromatograms were edited with the Chromas software and database similarity matching to identify the fungal strain was done using NCBI BLAST.

\section{Determination of secondary metabolites}

One plug (about $3 \mathrm{~mm}$ diameter) each of the Talaromyces flavus and that of the reference strain Aspergillus fumigatus (ATCC 3667) were individually inoculated into $15 \mathrm{~mL}$ of potato dextrose broth (PDA) prepared with dam water and nuclease-free water. The supplement of $25 \%$ PDA concentration was used to balance nutrients and liquidity of the broth. To determine the secondary metabolites produced by fungi in water, the media were incubated at $28{ }^{\circ} \mathrm{C}$ in a shaking incubator maintained at $150 \mathrm{r} / \mathrm{min}$. The samples were collected from the incubator in triplicate after $0 \mathrm{~h}, 72 \mathrm{~h}$ and 7 days (Table 1). The $\mathrm{pH}$ and conductivity were measured during the collection time. Solid phase extraction (SPE) procedure was used for the extraction of both polar and non-polar compounds. The Oasis ${ }^{\circledR}$ HLB $(3 \mathrm{~mL})$ cartridges (Hydrophilic Lipophilic Balance (HLB), Waters Oasis ${ }^{\circledR}$ sample extraction products) were used for the SPE 
procedure. Secondary metabolites were extracted from $10 \mathrm{~mL}$ of the broth media. The first step was to condition the solid phase adsorbed with $3 \mathrm{~mL}$ of $50 \% \mathrm{MeOH}-\mathrm{H}_{2} \mathrm{O}$, at the flow rate of approximately less than $3 \mathrm{~mL} / \mathrm{min}$. The samples were then introduced into the cartridge at the flow rate of about $8 \mathrm{~mL} / \mathrm{min}$. After sample loading the sample was washed with $3 \mathrm{~mL}$ of $5 \%$ methanol-water. Cartridges were then dried for more than 30 min under vacuum. The analyte was then eluted with $2 \times 3 \mathrm{~mL}$ of $50 \% \mathrm{MeOH}-\mathrm{H}_{2} \mathrm{O}$. The extracts were dried using nitrogen gas to near dryness at $30{ }^{\circ} \mathrm{C}$ under reduced pressure, and then re-dissolved in $2 \mathrm{~mL}$ of methanol and transferred to amber vials. It was then further evaporated to near dryness at $35{ }^{\circ} \mathrm{C}$ to concentrate the sample, and subsequently re-dissolved in $500 \mu \mathrm{L}$ of methanol-water (1:1).

Table 1. The sample treatments with reference strain and isolated fungi in nuclease-free water $(C W)$ and Dam water $(D W)$

\begin{tabular}{|c|c|c|c|c|c|c|c|c|}
\hline \multirow{2}{*}{$\begin{array}{c}\text { Fungi } \\
\text { Aspergillus } \\
\text { Fumigatus }\end{array}$} & \multirow{2}{*}{\begin{tabular}{|c|} 
Water \\
$\begin{array}{l}\text { Nuclease- } \\
\text { Free water }\end{array}$
\end{tabular}} & \multirow{2}{*}{\begin{tabular}{|c|}
$\begin{array}{c}\text { Treatments } \\
\text { (PDA) }\end{array}$ \\
$0 \%$ \\
\end{tabular}} & \multicolumn{3}{|c|}{ Incubation time } & \multicolumn{3}{|c|}{ Samples } \\
\hline & & & $0 \mathrm{~h}$ & $72 \mathrm{~h}$ & 7 days & $\begin{array}{c}\text { Oh-A-0\%- } \\
\text { CW }\end{array}$ & $\begin{array}{c}\text { 72h-A-0\%- } \\
\text { CW }\end{array}$ & D-A-0\%-CW \\
\hline $\begin{array}{l}\text { Aspergillus } \\
\text { Fumigatus }\end{array}$ & $\begin{array}{l}\text { Nuclease- } \\
\text { Freewater }\end{array}$ & $25 \%$ & $0 \mathrm{~h}$ & $72 \mathrm{~h}$ & 7 days & $\begin{array}{c}\text { Oh-A-25\%- } \\
\text { CW }\end{array}$ & $\begin{array}{l}72 \mathrm{~h}-\mathrm{A}- \\
25 \% \mathrm{CW}\end{array}$ & $\begin{array}{c}\text { 7D-A-25\%- } \\
\text { CW }\end{array}$ \\
\hline $\begin{array}{l}\text { Aspergillus } \\
\text { Fumigatus }\end{array}$ & Dam water & $0 \%$ & $0 \mathrm{~h}$ & $72 \mathrm{~h}$ & 7 days & $\begin{array}{c}\text { Oh-A-0\%- } \\
\text { DW }\end{array}$ & $\begin{array}{c}\text { 72h-A-0\%- } \\
\text { DW }\end{array}$ & $\begin{array}{c}\text { 7D-A-0\%- } \\
\text { DW }\end{array}$ \\
\hline $\begin{array}{l}\text { Aspergillus } \\
\text { Fumigatus }\end{array}$ & Dam water & $25 \%$ & $0 \mathrm{~h}$ & $72 \mathrm{~h}$ & 7 days & $\begin{array}{c}\text { 0h-A-25\%- } \\
\text { DW }\end{array}$ & $\begin{array}{l}72 \mathrm{~h}-\mathrm{A}- \\
25 \% \mathrm{DW}\end{array}$ & $\begin{array}{c}\text { 7D-A-25\%- } \\
\text { DW }\end{array}$ \\
\hline $\begin{array}{c}\text { Talaromyces } \\
\text { Flavus }\end{array}$ & $\mid \begin{array}{l}\text { Nuclease- } \\
\text { Freewater }\end{array}$ & $0 \%$ & $0 \mathrm{~h}$ & 72 & $7 \mathrm{~d} c$ & $\begin{array}{c}\text { Oh-T-0\%- } \\
\text { CW }\end{array}$ & 7-CW & $\begin{array}{c}\text { 7D-T-0\%- } \\
\text { CW }\end{array}$ \\
\hline $\begin{array}{c}\text { Talaromyces } \\
\text { Flavus }\end{array}$ & 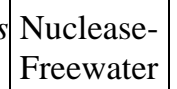 & $25 \%$ & $0 \mathrm{~h}$ & $72 \mathrm{~h}$ & 7 days & $\begin{array}{c}\text { 0h-T-25\%- } \\
\text { CW }\end{array}$ & $\begin{array}{c}\text { 72h-T-25\%- } \\
\text { CW }\end{array}$ & $\begin{array}{c}\text { 7D-T-25\%- } \\
\text { CW }\end{array}$ \\
\hline $\begin{array}{c}\text { Talaromyces } \\
\text { Flavus }\end{array}$ & Dam water & $0 \%$ & $0 \mathrm{~h}$ & $72 \mathrm{~h}$ & 7 days & $\begin{array}{c}\text { Oh-T-0\%- } \\
\text { DW }\end{array}$ & $\begin{array}{c}\text { 72h-T-0\%- } \\
\text { DW }\end{array}$ & $\begin{array}{c}\text { 7D-T-0\%- } \\
\text { DW }\end{array}$ \\
\hline $\begin{array}{c}\text { Talaromyces } \\
\text { Flavus }\end{array}$ & Dam & $25 \%$ & $0 \mathrm{~h}$ & 72 & 7 day & $\begin{array}{c}\text { 0h-T- } 25 \%- \\
\text { DW }\end{array}$ & \begin{tabular}{|c|} 
72h-T-25\%- \\
DW
\end{tabular} & $\begin{array}{c}\text { 7D-T-25\%- } \\
\text { DW }\end{array}$ \\
\hline $\begin{array}{l}\text { Without } \\
\text { Fungi }\end{array}$ & $\begin{array}{l}\text { Nuclease- } \\
\text { Freewater }\end{array}$ & $0 \%$ & $0 \mathrm{~h}$ & $72 \mathrm{l}$ & 7 days & $\begin{array}{c}\text { Oh-C-0\%- } \\
\text { CW }\end{array}$ & $\begin{array}{c}\text { 72h-C-0\%- } \\
\text { CW }\end{array}$ & $\begin{array}{c}\text { 7D-C-0\%- } \\
\text { CW }\end{array}$ \\
\hline $\begin{array}{l}\text { Without } \\
\text { Fungi }\end{array}$ & $\begin{array}{l}\text { Nuclease- } \\
\text { Freewater }\end{array}$ & $25 \%$ & $0 \mathrm{~h}$ & $72 \mathrm{~h}$ & 7 days & $\begin{array}{c}\text { 0h-C-25\%- } \\
\text { CW }\end{array}$ & \begin{tabular}{|c|} 
72h-C-25\%- \\
$\mathrm{CW}$
\end{tabular} & $\begin{array}{c}\text { 7D-C-25\%- } \\
\text { CW }\end{array}$ \\
\hline $\begin{array}{l}\text { Without } \\
\text { Fungi }\end{array}$ & Dam water & $0 \%$ & $0 \mathrm{~h}$ & $72 \mathrm{~h}$ & 7 days & $\begin{array}{c}\text { 0h-C-0\%- } \\
\text { DW }\end{array}$ & $\begin{array}{c}\text { 72h-C-0\%- } \\
\text { DW }\end{array}$ & $\begin{array}{l}\text { 7D-C-0\%- } \\
\text { DW }\end{array}$ \\
\hline $\begin{array}{l}\text { Without } \\
\text { Fungi }\end{array}$ & Dam water & $25 \%$ & $0 \mathrm{~h}$ & $72 \mathrm{~h}$ & 7 days & $\begin{array}{c}\text { 0h-C-25\%- } \\
\text { DW }\end{array}$ & \begin{tabular}{|c|} 
72h-C-25\%- \\
DW
\end{tabular} & $\begin{array}{c}\text { 7D-C-25\%- } \\
\text { DW }\end{array}$ \\
\hline
\end{tabular}

\section{Instrumental analysis using LC-QTOF-MS (Bruker, Bremen, Germany)}

The secondary metabolites excreted by fungi were separated in a reversed-phase chromatography (RPC) procedure, using a Waters Acquity UPLC BEH C18 $1.7 \mu \mathrm{m}$ 2.1 X $100 \mathrm{~mm}$ column. After separation, secondary metabolites were analysed using LC-QTOF-MS (Impact II system). The analysis was done under positive mode. Secondary metabolites were Analytes of interest eluted with mobile phase A (water acidified with $0.1 \%$ formic acid) and B (LC-MS grade methanol acidified with $0.1 \%$ formic acid). The sample aliquot of $10 \mu \mathrm{L}$ was injected into the $\mathrm{C} 18$ column and eluted using the gradient method at the speed of $0.2 \mathrm{~mL} / \mathrm{min}$. 


\section{Statistical analysis}

The influence of nutrients, water type and fungal species on the biosythesis of secondary metabolites was evaluated using principal component analysis from Soft Independent Modelling of Class Analogy (SIMCA) software. The samples were presented in triplicate for each treatment in dam water and the other set in nuclease free water. Related secondary metabolites produced by Talaromyces flavus and Aspergillus fumigatus (ATCC 36607) during incubation process were assessed in PCA plots.

\section{Results}

\section{Identification of fungal species}

The pure fungal species of Talaromyces flavus was isolated from dam water. This fungus is known as an endophyte however in this study was isolated and identified from a water sample. The PCR results (Figure 2) shows a DNA amplification of a PCR band which corresponds to an approximately $500 \mathrm{bp}$. The PCR bands indicated the presence of fungal DNA in water samples (Figure 2). The fungal diagnostic primer pair ITS1F and ITS4 was used in the PCR to confirm the presence of fungal DNA. The PCR product was sent for Sanger sequencing and the obtained sequence was compared with the available sequences on the NCBI database by basic local alignment search tool (BLAST). In addition to the identification done by sequence analysis of the ITS region, the fungal species was also identified morphologically.
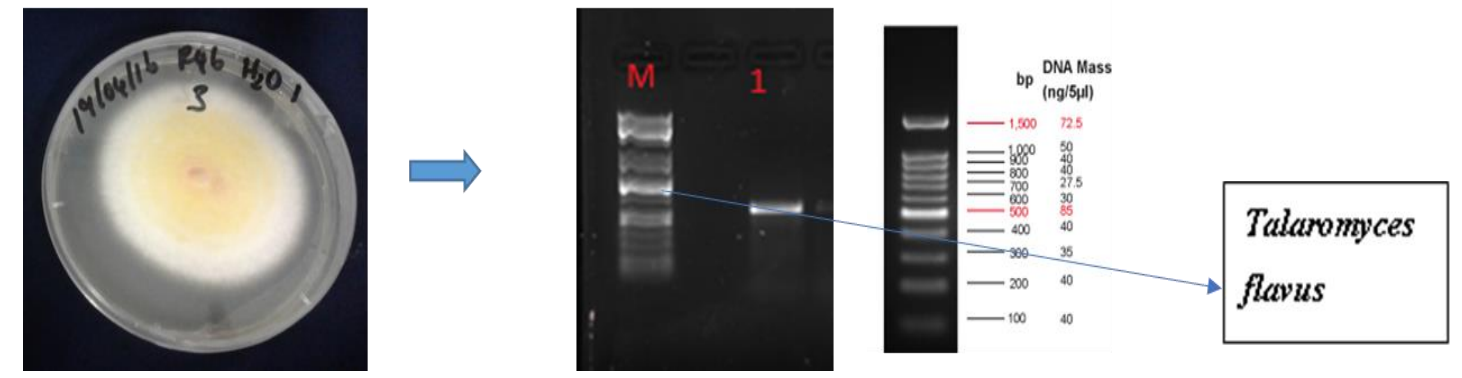

Figure 2. The pure fungal cultures grown in PDA. Talaromyces flavus Fungal DNA bands in $1 \%$ agarose gel. (M) Ladder, (1) water sample 1

\section{Effect of physical parameters}

Upon the introduction of the fungal plugs of $T$. flavus and A. fumigatus, the growth media conditions were found to change during the incubation period of 7 days. The changes in the media, $\mathrm{pH}$, conductivity, and incubation conditions have a major role on the production of secondary metabolites. The incubation conditions depend on ecology and physiology of various filamentous fungi. In this study the PDA broth media were incubated at $28{ }^{\circ} \mathrm{C}$, which is the recommended temperature for Talaromyces, Aspergillus and Penicillium species (Zhu et al., 2014). The influence of PDA nutrient was found to be proportional with the $\mathrm{pH}$ of the broth samples. In the absence of nutrients, the $\mathrm{pH}$ decreases gradually, while a sharp decrease in $\mathrm{pH}$ was observed with an excess amount of nutrients (Figure 3). This finding indicates the role played by aquatic fungi in that they utilise the nutrients available in the aquatic environment. The 
nutrients promote high fungal growth which leads to the production of various secondary metabolites.

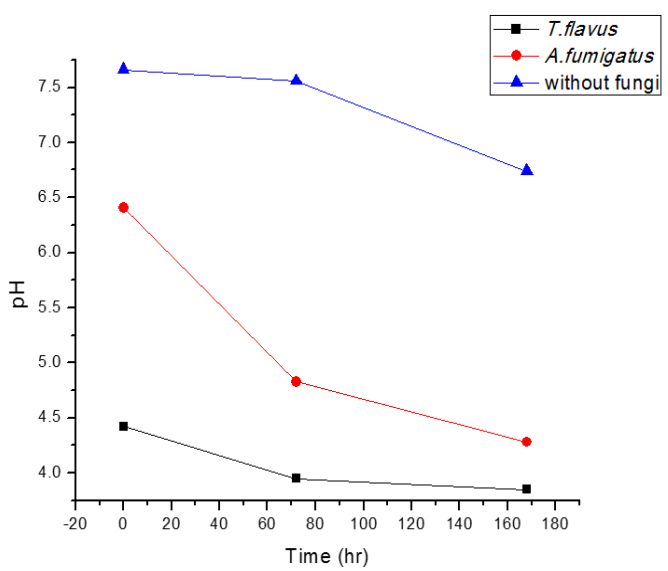

A

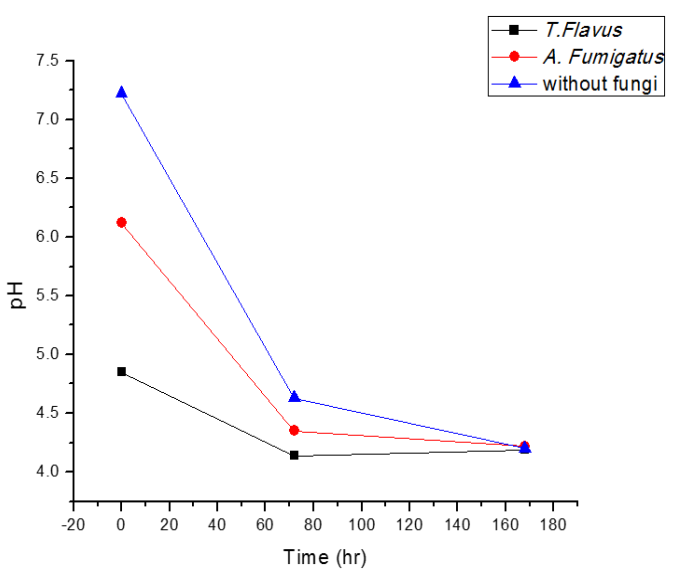

B

Figure 3. The relationship between the nutrient level and $p H$ over the incubation period of 7 days. (A) absence of nutrients, (B) excess amount of nutrients

The decomposition of organic material by fungi has an impact on the nutrient levels in water bodies (Leigh et al., 2009). The trend of the change in $\mathrm{pH}$ and conductivity over an incubation period of 7 days is similar for water samples with the fungal plug (Figure 4A-D). The growth media without the fungal plug showed a different trend (Figure 4E,F); however, the $\mathrm{pH}$ of dam water after an incubation period of 7 days was found to be more acidic compared to nuclease-free water, because of the other compounds present in the dam water. The amount of dissolved material is proportional to the electrical conductivity of water. The conductivity was found to be lower in nuclease-free water without the fungal plug because of the lower concentration of ions in the nuclease-free water. The $\mathrm{pH}$ was found to decrease between $0 \mathrm{~h}$ and $72 \mathrm{~h}$ because the fungi consumed many of the nutrients, but the trend changed after $72 \mathrm{~h}$; the $\mathrm{pH}$ increases are due to the depletion of nutrients (Figure 4A). During the culture period, the $\mathrm{pH}$ trend was found to be negatively correlated to that of conductivity of water. The starting $\mathrm{pH}$ for dam water was more acidic compared to that of the nuclease-free water with the plug of fungi.

The studies recorded maximum extracts after the incubation period of 7 days. Some changes in the profiling of secondary metabolites were observed after incubating for $72 \mathrm{~h}$ and 7 days. There was an increase in the number of compounds released by the isolated fungi (T. flavus) and reference strain (A. fumigatus) in the media with a higher nutrient level of PDA broth. The SIMCA PCA plots demonstrated the change in the orientation of secondary metabolites during the incubation process. At $0 \mathrm{~h}$ there was no significant difference in the natural products produced in dam water and nuclease-free water at various nutrient levels (Figure 5). However, the variable with similar properties are coming together.

After an incubation period of $72 \mathrm{~h}$, the groupings of similar compounds were observed in three clusters (Figure 6). The samples without fungi are grouped together and the other two clusters are grouped related to nutrient level. Cluster 1 representing the samples treated with 25\% PDA nutrients in the presence of Talaromyces flavus and 
Aspergillus fumigatus (ATCC 36607). The samples treated with PDA are distinguishable with the sample without treatment, that indicates the difference in compounds produced by fungi. Second group (2) showed the samples without treatment forming a cluster because of similar chemical properties and the minimal secondary metabolites produced under this condition. The control samples with and without PDA treated grouped together to form the third cluster 3.
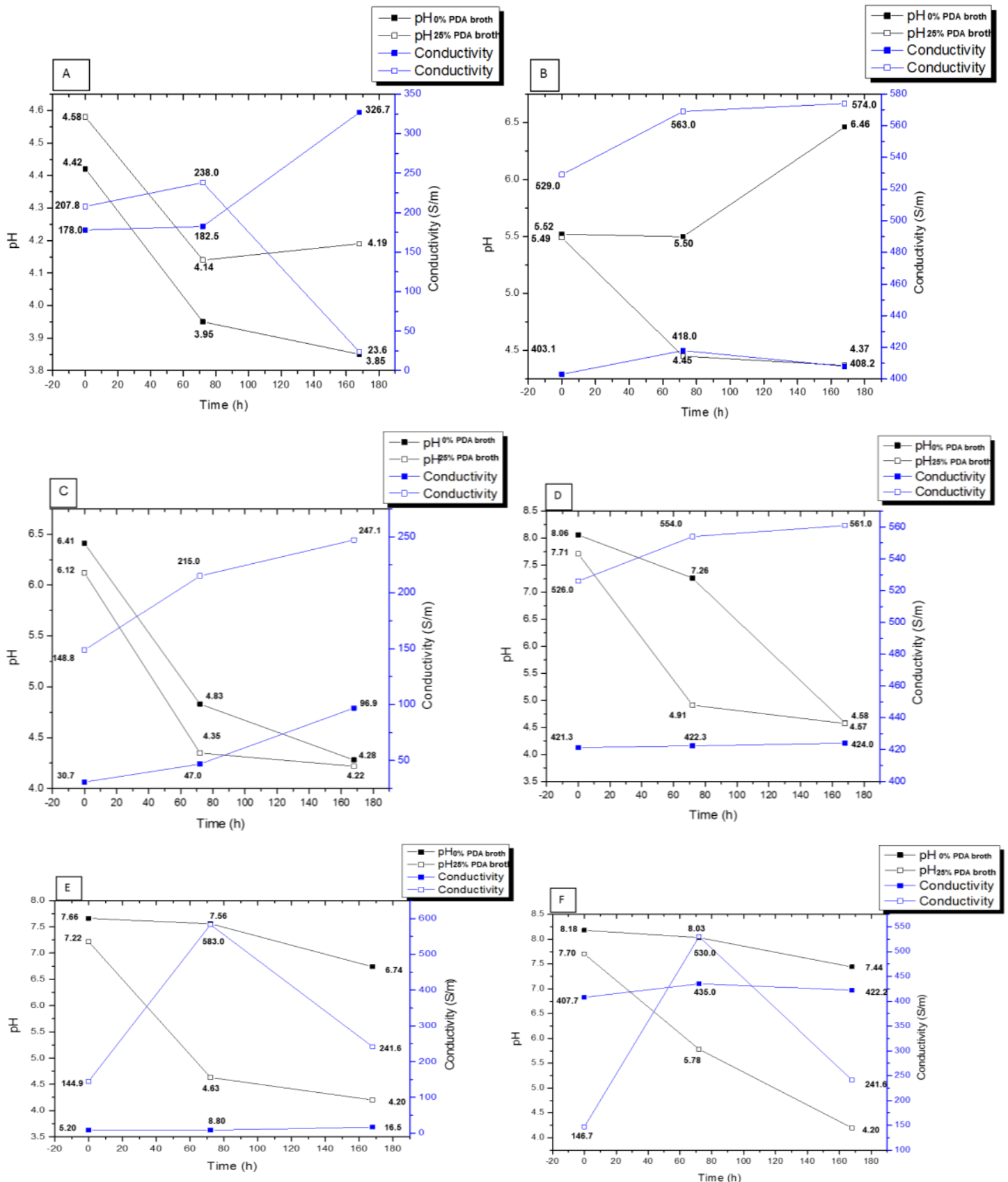

Figure 4. The effect of fungi on the $\mathrm{pH}$ and conductivity of the growth media: (A) T. flavus in nuclease-free water; (B) T. flavus in dam water; $(C)$ A. fumigatus in nuclease-free water; $(D) A$. fumigatus in dam water; $(E)$ without fungi in nuclease-free water; and $(F)$ without fungi in dam 


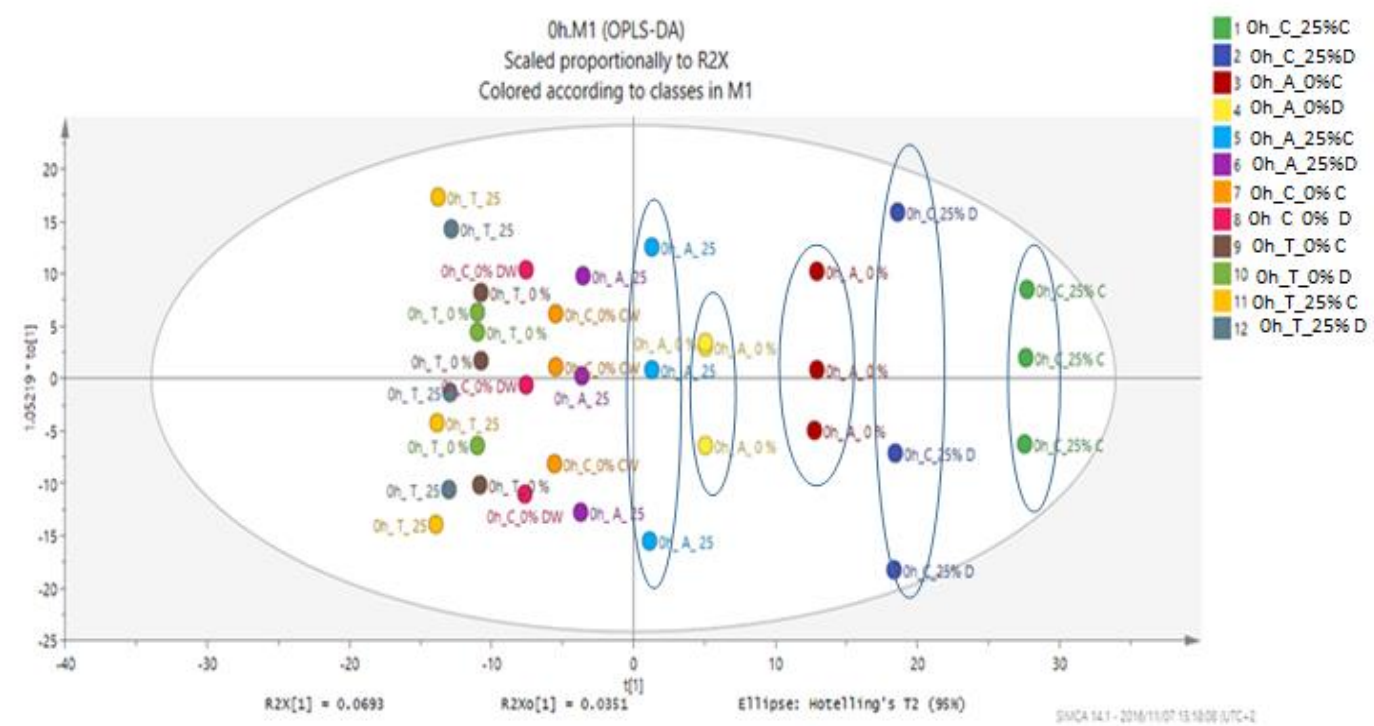

Figure 5. The principal component analysis (PCA) scatter plots of secondary metabolites produced by fungi after $0 \mathrm{~h}$ : (1) without fungi at $25 \%$ PDA in nuclease-free water; (2) without fungi at $25 \%$ PDA in dam wate0r; (3) A. fumigatus at $0 \%$ PDA in nuclease-free water; (4) A. fumigatus at $0 \%$ PDA in dam water; (5) A. fumigatus at $25 \%$ PDA in nuclease-free water; (6) A. fumigatus at $25 \%$ PDA in dam water; (7) without fungi at $0 \%$ PDA in nuclease-free water; (8) without fungi at $0 \%$ PDA in dam water; (9) T. flavus at O\% PDA in nuclease-free water;

(10) T. flavus at $0 \%$ PDA in dam water; (11) T. flavus at $25 \%$ PDA in nuclease-free water; and (12) T. flavus at $25 \%$ PDA in dam water

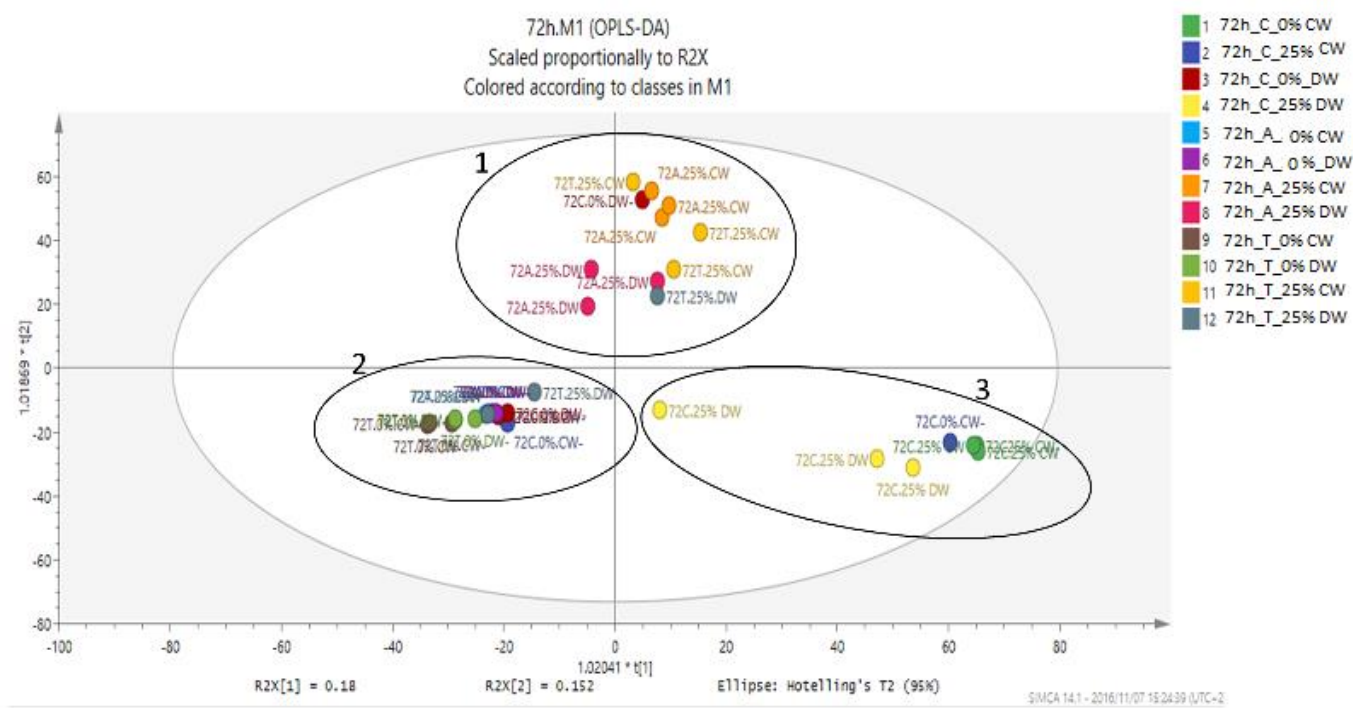

Figure 6. The principal component analysis (PCA) scatter plots of secondary metabolites produced by fungi after $72 \mathrm{~h}$ : (1) without fungi at $0 \%$ PDA in nuclease-free water; (2) without fungi at $25 \%$ PDA in nuclease-free water; (3) without fungi at $0 \%$ PDA dam water. (4) without fungi at $25 \%$ PDA in dam water. (5) A. fumigatus at $0 \%$ PDA in nuclease-free water; (6) A. fumigatus at $25 \%$ PDA in dam water (7) A. fumigatus at $25 \%$ PDA in nuclease-free water; (8) A. fumigatus at $25 \%$ PDA in dam water (9) T. flavus at $0 \%$ PDA in nuclease-free water; (10) T. flavus at $0 \%$ PDA in dam water; (11) T. flavus at $25 \%$ PDA in nuclease-free water; and (12) T. flavus at $25 \%$ PDA in dam water 
The distribution of secondary metabolites after 7 days showed a significant difference within the groups of samples; however, the stress conditions start to increase when the nutrient levels are no longer high enough to be used by fungi to produce new secondary metabolites (Figure 7). Longer incubation showed more interesting results; cluster 1 remained the same after 7 days for samples with fungal plug and 25\% PDA treatment. The reorientation observed for cluster $\mathbf{2}$ and $\mathbf{3}$ there is an exchange of quadrants. In addition, group $\mathbf{2}$ showed all the samples without treatment such as control and the samples with fungal plug of Talaromyces flavus and Aspergillus fumigatus (ATCC 36607). The third cluster is the grouping of samples without fungal plug in the present of PDA treatment.

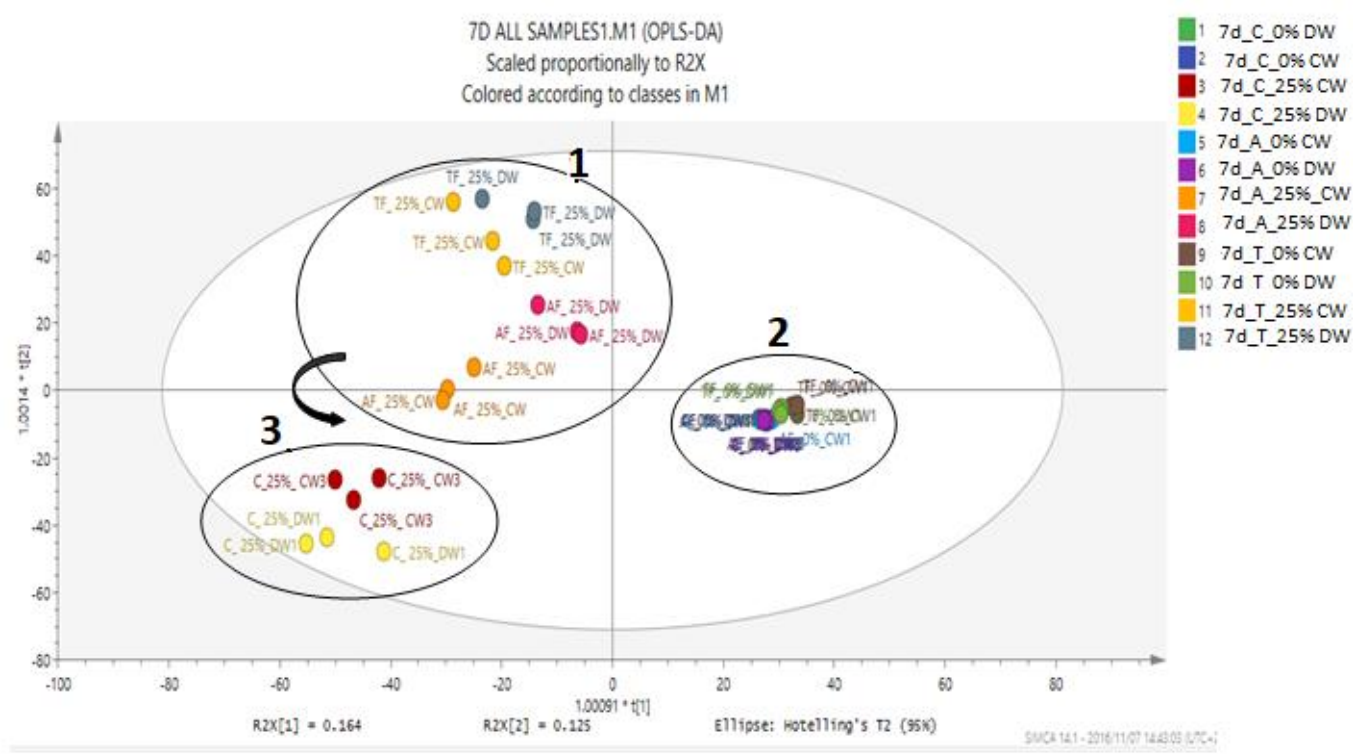

Figure 7. The principal component analysis (PCA) scatter plots of secondary metabolites produced by fungi after 7 days: (1) without fungi at $0 \%$ PDA in dam water; (2) without fungi at $0 \%$ PDA in nuclease-free water; (3) without fungi at $25 \%$ PDA in nuclease-free water; (4) without fungi at $25 \%$ PDA dam water; (5) A. fumigatus at $0 \%$ PDA in nuclease-free water; (6) A. fumigatus at $0 \%$ PDA in dam water; (7) A. fumigatus at $25 \%$ PDA in nuclease-free water; (6) A. fumigatus at $25 \%$ PDA in dam water; (8) A. fumigatus at $25 \%$ PDA dam water; (9) T. flavus at $0 \%$ PDA in nuclease-free water; (10) T. flavus at $0 \%$ PDA in dam water; (11) T. flavus at $25 \%$ PDA in nuclease-free water; and (12) T. flavus at $25 \%$ PDA in dam water

Figures 8(1) and (3) show the LC/MS chromatograms only for the secondary metabolites produced by $T$. flavus and A. fumigatus in nuclease-free water. The different chemical profile of secondary metabolites shows that the nutrients influenced the qualitative production of secondary fungal metabolites. As seen on the LC/MS chromatogram of the sample with high nutrients (Figures $8(2)$ and (4)), there are new peaks between retention times of 4 to $8 \mathrm{~h}$ with higher intensity. In addition, the incubation period seems to also play a major role in the number of natural products produced by fungi. Comparisons of the profiling chromatogram at $72 \mathrm{~h}$ with 7 days' reveal that the fingerprint pattern is the same; however, the intensity is higher after 7 days. New peaks appeared in the chromatogram of the sample with a high nutrient level; this illustrates the important role played by nutrients in the biosynthesis of 
secondary metabolites. It should be noted that the new peaks from dam water could be secondary metabolites, degraded compounds or bio-transformed products due to fungal activities.
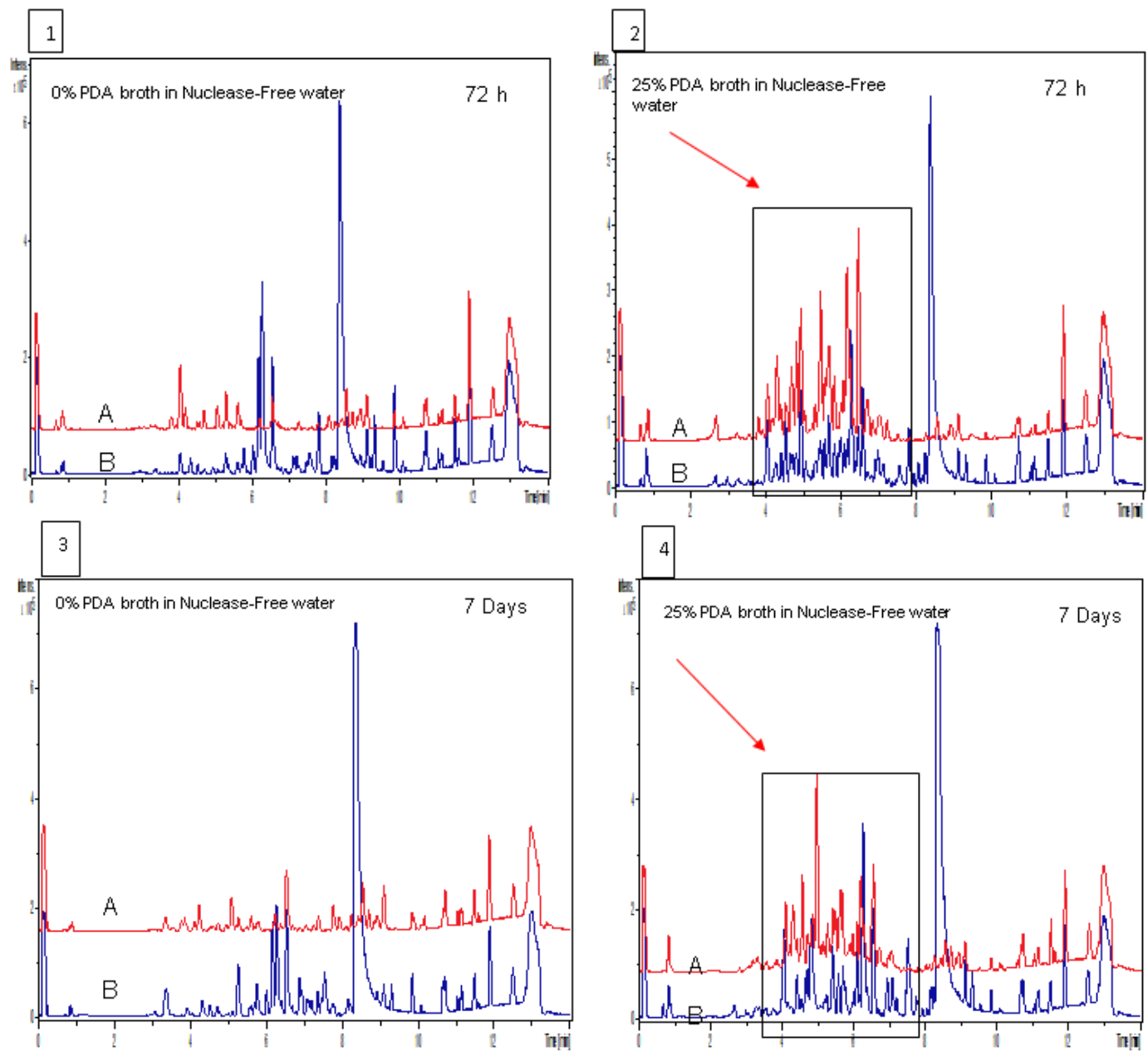

Figure 8. The LC-MS chromatograms of extracellular secondary metabolites produced by fungi in nuclease-free water: (A) T. flavus; and (B) A. fumigatus

\section{Discussion}

The ITS primers used in this study have been found to have higher sensitivity and produce PCR bands which showed clear amplification of approximately 500 nucleotides fragments (Ferrer et al., 2001). Both the morphological identification and the sequence analysis of the ITS region pointed to Talaromyces flavus as the isolated fungus from Roodeplaat Dam.

Talaromyces flavus is commonly found in the soil, plants and major crops such as cotton and potato. It is known as an antagonist that has been used in biological control of some soil-borne and plant pathogens (Ayer et al., 1990; Zhong, 2009; Naraghi et al., 2012). Talaromyces flavus has been studied extensively as a biological control of agricultural pathogens by various processes, including parasitism, competition for nutrients and antibiosis (Cordell and Cordell, 2016; Fravel and Roberts, 2016). This fungus is unique and important compared to others due to its extreme tolerance to heat. 
In this study T. flavus was isolated from the dam water, while most of the other studies have been identifying this fungus from soil, plants and agricultural commodities.

Screening of T.flavus secondary metabolites produced in the artificial media was investigated using dam water and nuclease-free water. As the incubation time increased the growth media were found to be more acidic during the incubation period of 7 days; that was because of the change of nutrients in water. The spike of T. flavus and reference strain A. fumigatus (ATCC 36607) caused the $\mathrm{pH}$ to drop, which means that the fungi utilise dissolved oxygen to break down organic matter, leading to an increase in the amount of dissolved material, hence higher electrical conductivity. However, the conductivity of the broth media prepared with nuclease-free water without the fungal plug showed lower conductivity compared to all other samples. That was expected since nuclease-free water does not contain many compounds compared to the dam water which has a more complex composition.

The availability of aquatic fungal species in surface water mostly depends on the chemical and physical properties of water, since they survive under various favourable conditions (Thakur, 2009). In this study it has been proven that fungal growth influences the quality of the water by changing physical parameters. The $\mathrm{pH}$ was found to decrease gradually during the incubation period and the lower $\mathrm{pH}$ levels increase the risk of mobilised toxic metals that can be absorbed by aquatic organisms; the change in pH affects many sensitive species in water ecosystems (Barnes et al., 1998). The higher the $\mathrm{pH}$, the higher the solubility effect of some other compounds and elements, which results in the formation of toxic compounds or chemicals that can be easily absorbed by aquatic life (Barrie and Georgii, 1976). Casellato and Said (2006) reported a large number of conidia after 7 days compared to 10 days; this observation is in line with the results reported in this study. The prolonged incubation period increases the stressful environment for microorganisms especially when there are not enough nutrients for survival. The results of this study corroborate the results obtained by Ayer and Racok (1990), who stated that during the incubation period of 5 days the $\mathrm{pH}$ of the broth with T. flavus decreases.

Cultivation conditions strongly influence the production of bioactive fungal compounds. Parameters such as nutrients, incubation period, $\mathrm{pH}$ and temperature are important in the production of secondary metabolites. The study conducted by Schulz et al. on the influence of culture conditions on the production of secondary metabolites by fungi reported observations which are similar to those reported in this present study (Schulz et al., 2008). Both studies found $28{ }^{\circ} \mathrm{C}$ as a recommended incubation temperature for conidia production.

The known secondary metabolites produced by A. fumigatus were successfully extracted from both media prepared with dam water and nuclease-free water, indicating that the method for the extraction of secondary metabolites worked well. There is a possibility that some other compounds which are not secondary metabolites may be observed in the dam water; due to biotransformation or biodegradation of substrates in the dam water. In the case of nuclease-free water, the significant difference observed in the PCA plot was without any doubt because of the secondary metabolites produced by the fungi. Significant differences in metabolites were observed in the samples of dam water with varying nutrient levels; the results clearly showed the role of nutrients in the biosynthesis of natural products. The presence of nutrients triggers numerous activities and processes in the complex matrix of dam water because of the many compounds involved. 


\section{Recommendations}

- Identification of the secondary fungal metabolites is required for the discovery of novel drugs and useful compounds in pharmaceutical industries. In addition, the proposed biosynthesis mechanism for production of secondary metabolites needs to be explored as it may offer new opportunities for extraction.

- To date, only a few studies are focused on the biological role of aquatic fungi as well as their secondary metabolites in freshwater aquatic ecosystems. That might contribute to the lack of some useful drugs for treatment of various infections or else the toxins that can be harmful to humans and animals. Further research is required for the discovery of novel metabolites from fungi, as well as focusing on removal of toxigenic fungal species from water sources.

\section{Conclusions}

This study investigated the conditions that favour the biosynthesis of various classes of secondary metabolites by $T$. flavus and A. fumigatus. Every fungal species has its own gene responsible for the synthesis of secondary metabolites; however, the optimum conditions for mycelium growth do not determine the biosynthesis of secondary metabolites. No direct relationship could be established between the rate of fungal growth and secondary metabolite production; it occurs as a specific response to several environmental stress factors, including biotic and abiotic elicitors (the chemical compounds from abiotic and biotic sources that can stimulate stress responses in plants). The regulator gene can be up-regulated or down-regulated under conditions conducive to growth, such as carbon source, nitrogen source, oxidative stress, temperature, and lower $\mathrm{pH}$. The extraction methods was successfully since the known group of secondary metabolites was identified from reference strain (A.fumigatus ATCC 36607) culture.

Acknowledgments. We thank Mr Garland More with his assistant on SIMCA software. We are very grateful to the following organizations: University of South Africa, National Research Foundation and Institute for Nanotechnology and Water Sustainability for funding this research.

Compliance with Ethical Standards. The authors declare that there is no conflict of interests regarding the publication of this paper and that the research data included in the manuscript do not involve either human participants or animals.

\section{REFERENCES}

[1] Ayer, W. A., Racok, J. S. (1990): The metabolites of Talaromycesflavus: Part 1. Metabolites of the organic extracts. - Canadian Journal of Chemistry 68: 2085-2094.

[2] Ayer, W. A., Racok, J. S. (1990): The metabolites of Talaromycesflavus: Part 2. Biological activity and biosynthetic studies. - Canadian Journal of Chemistry 68: 20952101.

[3] Barrie, L. A., Georgii, H. W. (1976): An Experimental Investigation of the Absorption of Sulphur Dioxide by Water Drops Containing Heavy Metal Ions. - Atmospheric Environment 10: 743-749.

[4] Calvo, A. M., Richard, A. W., Bok, J. W., Keller, N. P. (2002): Relationship between Secondary Metabolism and Fungal Development. - Microbiology. Mol. Biol 66: 447459. 
[5] Casellato, B., Said, S. (2006): The influence of culture conditions on the biosynthesis of secondary metabolites by Penicillium verrucosum Dierck. - Microbiological Research 161(3): 273-280.

[6] Cray, J. A., Connor, C., Stevenson, A., Houghton, D. R., Rangel, E. N., Cooke, L. R., Hallsworth, J. E. (2016): Biocontrol agents promote growth of potato pathogens, depending on environmental conditions. - Applied microbiology, Microbial Biotechnology 9: 330-354.

[7] El-hasan, A., Walker, F., Schone, J., Buchenauer, H. (2009): Detection of viridiofungin A and other antifungal metabolites excreted by Trichoderma harzianum active against different plant pathogens. - Eur Journal Plant Pathol 124: 457-470.

[8] Ferrer, C., Colom, F., Frases, S., Mulet, E., Abad, J., Alio, J. (2001): Detection and Identification of Fungal Pathogens by PCR and by ITS2 and 5.8S Ribosomal DNA Typing in Ocular Infections Detection and Identification of Fungal Pathogens by PCR and by ITS2 and 5.8S Ribosomal DNA Typing in Ocular Infections. - Journal of clinical microbiology 39: 2873-2879.

[9] Fravel, D. R., Adams, P. B. (1987): Estimation of United States and World Distribution of Talaromyces flavus. - Mycologia 78: 684-686.

[10] Fravel, D. R., Roberts, D. P. (2016): In situ Evidence for the role of Glucose oxidase in the biocontrol of verticillium wilt by Talaromyces flavus in situ Evidence for the Role of Glucose Oxidase in the Biocontrol of Verticillium Wilt by Talaromyces flavus. Biocontrol Science and Technology 1: 91-99.

[11] Gaden, E. L. (2000): Fermentation Process Kinetics. - Journal of Biochemical and microbiology technology and engineering 67: 413-419.

[12] Hernández-Carlos, B., Gamboa-Angulo, M. M. (2011): Metabolites from freshwater aquatic microalgae and fungi as potential natural pesticides. - Phytochemistry Reviews 10: 261-286.

[13] Imhoff, J. F. (2016): Natural Products from Marine Fungi - Still an Underrepresented Resource. - Mar. Drugs 14: 2-19.

[14] Khan, A. A., Bacha, N., Ahmad, B., Lutfullah, G., Farooq, U., Cox, R. J. (2014): Fungi as chemical industries and genetic engineering for the production of biologically active secondary metabolites. - Asian Pacific Journal of Tropical Biomedicine 4: 859-870.

[15] Leigh, J., Hodge, A., Fitter, A. H. (2009): Arbuscular mycorrhizal fungi can transfer substantial amounts of nitrogen to their host plant from organic material. - New Phytologist 181: 199-207.

[16] Naraghi, L., Heydari, A., Rezaee, S., Razavi, M. (2012): Biocontrol Agent Talaromyces flavus Stimulates the Growth of Cotton and Potato. - Journal of plant growth Regul. 31: 471-477.

[17] Schulthess, B., Ledermann, R., Mouttet, F., Zbinden, A., Bloemberg, G. V., Bottger, E. C., Hombach, M. (2014): Use of the Bruker MALDI Biotyper for identification of molds in the clinical mycology laboratory. - Journal of clinical microbiology 52: 2797-803.

[18] Schulz, B., Draeger, S., Cruz, T. E., Rheinheimer, J., Siems, K., Loesgen, S., Bitzer, J., Schloerker, O., Zeeck, A., Kock, I., Hussain, H., Dai, J., Krohn, K. (2008): Screening strategies for obtaining novel, biologically active, fungal secondary metabolites from marine habitats. - Botanica Marina 51: 219-234.

[19] Singh, M. P., Leighton, M. M., Barbieri, L. R., Roll, D. M., Urbance, S. E., Hoshan, L., McDonald, L. A. (2010): Fermentative production of self-toxic fungal secondary metabolites. - Journal of Ind Microbiology Biotechnol 37: 335-340.

[20] Swathi, J., Narendra, K., Sowjanya, K. M., Satya, A. K. (2013): Marine fungal metabolites as a rich source of bioactive compounds. - African Journal of Biochemistry Research 7: 184-196.

[21] Thakur, A. S. B. (2009): Mycological Society of America Survival of Some Aquatic Hyphomycetes under Dry Conditions. - Mycologia 69: 843-845. 
[22] Zhao, J., Zhou, L., Wang, J., Shan, T., Zhong, L., Liu, X., Gao, X. (2010): Endophytic fungi for producing bioactive compounds originally from their host plants. - In: Applied microbiology and microbial biotechnology, pp. 567-576.

[23] Zhong, J. J., Xiao, J. H. (2009): Secondary Metabolites from Higher Fungi: Discovery, Bioactivity, and Bioproduction. - Adv Biochem Eng Biotechnol. 113: 79-150.

[24] Zhu, Y., Yao, L., Jiao, R., Lu, Y., Tan, R. (2014): Bioresource Technology Enhanced production of Fumigaclavine $\mathrm{C}$ in liquid culture of Aspergillus fumigatus under a twostage process. - Bioresource technology 152: 162-168. 